\title{
SARS-CoV-2 pandemic and the cardiovascular system: What the non-cardiologist needs to know
}

\author{
Authors: Gershan K Davis, ${ }^{A}$ Ahmed Adlan, ${ }^{B}$ Jacek Majewski ${ }^{C}$ and Bassem Ibrahim ${ }^{D}$
}

\begin{abstract}
There has been the need to make major modifications to the way cardiology is practised in light of the COVID-19 pandemic. There has also been the need to recognise the complex cardiovascular manifestations and complications of COVID-19. In this article we provide guidance on the management of cardiac patients without COVID-19 in the current pandemic as well as patients with cardiac disease and COVID-19 and patients with cardiac complications of COVID-19. There is also a focus on indications and interpretation of commonly performed cardiac investigations in the setting of COVID-19. References are included from a number of specialist societies and groups.
\end{abstract}

KEYWORDS: Coronavirus, heart, arrhythmias, chest infection, COVID-19

DOI: $10.7861 /$ clinmed.2020-0158

\section{Introduction}

The UK is affected by the novel coronavirus disease 2019 (COVID-19) due to the global spread of the severe acute respiratory syndrome coronavirus 2 (SARS-CoV-2).

We will discuss in this article relevant advice in four situations that the practicing physician may face in relation to COVID-19 and cardiovascular disease (CVD) in the current pandemic:

> the patient without COVID-19 but with CVD

$>$ differentiating COVID-19 symptoms from a presentation of CVD

$>$ the patient with COVID-19 and pre-existing CVD

$>$ the patient with COVID-19 who has developed cardiovascular complications.

A summary is provided in Box 1.

The present article is based on recommendation and guidelines from NHS England and the relevant British cardiac societies,


Central Lancashire, Preston UK and North Cumbria Integrated Care NHS Foundation Trust, Cumbria, UK; ${ }^{B}$ post-CCT cardiology fellow in electrophysiology and devices, Wythenshawe Hospital, Manchester, UK; ' Consultant cardiologist, North Cumbria Integrated Care Foundation Trust, Cumbria, UK, and honorary senior lecturer, University of Central Lancashire; ${ }^{D}$ consultant cardiologist, North Cumbria Integrated Care NHS Foundation Trust, Cumbria, UK including the British Cardiovascular Society (BCS), British Cardiovascular Interventional Society (BCIS), British Heart Rhythm Society (BHRS), Association for Inherited Cardiac Conditions, British Society of Heart Failure (BSH) and British Society of Echocardiography (BSE). Clinicians, however, are advised to refer to their local hospital/trust policies in the first instance.

\section{The patient without COVID-19 but with} cardiovascular disease

The management of these patients is aimed towards limitation of face-to-face contact and avoidance of non-urgent procedures. To this end, patients booked into outpatient clinics and on elective waiting lists will be reviewed by the relevant consultant cardiologist. Where possible, outpatient clinics are being conducted virtually, with only essential face-to-face appointments being carried out. Similarly, new referrals are triaged, and patients seen or deferred according to clinical urgency. Unless otherwise directed, referrals to outpatient services should continue; however, clinicians should be aware that appointments may be delayed and the availability of cardiac diagnostics services may be limited. All patients on elective waiting lists will be triaged and their procedures arranged/deferred as appropriate. In most cardiac tertiary centres, the capacity for cardiac procedures will be significantly reduced, with most centres offering urgent coronary intervention and pacing.

Acute coronary syndrome and out-of-hospital cardiac arrest

Primary percutaneous coronary intervention (PCI) remains the gold standard of care for patients with ST-elevation myocardial infarction (STEMI).

In non-ST-elevation MI (NSTEMI), coronary angiography may still be considered, particularly in the presence of high-risk features (ie troponin elevation $>5$ times upper limit of normal, dynamic electrocardiography changes, unstable symptoms despite treatment, haemodynamic instability, shockable cardiac arrest). Patients with NSTEMI or unstable angina without high risk features (eg small troponin rise, no ECG changes) or where disruptions to transport services results in significant delays in interhospital transfer ( $>5$ days) may be treated with optimal medical management and coronary angiography deferred as an outpatient.

For out-of-hospital cardiac arrest, only patients with clear ST elevation and no significant co-morbidities or other features suggesting a poor outcome should be transferred to a primary PCI centre. 


\section{Box 1. Summary of advice relating to cardiovascular disease management during COVID-19}

\section{Patients without COVID-19 but with cardiovascular disease}

Services are being restructured to limit face-to-face contact and avoid non-urgent procedures

Virtual clinics will replace outpatients and only essential face-to-face appointments will continue

Elective cardiac procedures will be deferred or scheduled based on urgency

Referrals to outpatient services should continue, although appointments may be delayed

Coronary angiography in ACS will be performed or delayed according to clinical urgency (eg primary PCI performed in STEMI, delayed in NSTEMI with

low risk features)

Optimisation of medications is key in ACS

PCI may be preferred over cardiac surgery in high-risk ACS with multivessel disease

Acute pacing will be available in most centres but reserved for high risk (eg high-grade AVB, severe symptomatic SND and long pauses)

Defibrillators (+/-CRT) will be reserved for secondary prevention (eg SCD survivors, ventricular arrhythmias with haemodynamic compromise)

Remote device checks and monitoring will be utilised where possible

Only urgent catheter ablations will be carried out (eg VT ablation in VT storm, pre-excited AF in WPW, atrial arrhythmias causing heart failure)

ACEi/ARBs should not be discontinued due to COVID-19 concerns

BNP testing in suspected heart failure can help prioritise patients needing urgent assessment

\section{Differentiating COVID-19 symptoms from a presentation of CVD}

Recognise that there is an overlap in clinical features of COVID-19 and cardiac diseases

Avoid (if possible) auscultation of the chest in patients with symptoms suggestive of COVID-19

Recognise that Brugada syndrome may be unmasked by fever in COVID-19, and although this is a rare condition there are important implications for management

The patient with COVID-19 and pre-existing CVD

There is a high prevalence of CVD in COVID-19 patients

CVD is associated with worse outcomes (ie increased need for intensive care, higher mortality)

The patient with COVID-19 who has developed cardiovascular complications

Heart failure is a common finding in patients with COVID-19, and carries a worse prognosis

Left ventricular systolic dysfunction may be pre-existing, or a result of COVID-19-induced myocarditis, or of inflammatory-mediated cardiomyopathy

Patients with left ventricular systolic dysfunction are at higher risk of needing mechanical ventilation

Elevated serum troponin and BNP confers a worse prognosis

Primary PCI remains the gold standard of care for STEMI in patients with COVID-19 but thrombolysis can be considered in selected cases

TTE can be considered in patients with COVID-19 but TOE should be avoided

Arrhythmias are common in COVID-19

PPM is generally deferred, but in unstable cases temporary pacing can be considered

Emerging drugs (eg lopinavir, hydroxychloroquine) carry pro-arrhythmia risks and may necessitate monitoring

$\mathrm{ACE} \mathrm{i}=$ angiotensin converting enzyme inhibitor, $\mathrm{ACS}=$ acute coronary syndrome, $\mathrm{AF}=$ atrial fibrillation, $\mathrm{ARB}=$ angiotensin receptor blocker, $\mathrm{AVB}=$ atrioventricular block, BNP = brain natriuretic peptide, CRT = cardiac resynchronisation therapy, CVD = cardiovascular disease, NSTEMI = non ST elevation myocardial infarction, $\mathrm{PCI}=$ percutaneous coronary intervention, PPM = permanent pacemaker, SCD = sudden cardiac death, SND = sinus node dysfunction, STEMI = ST elevation myocardial infarction, TTE $=$ transthoracic echocardiography, TOE = transoesophageal echocardiography, VT = ventricular tachycardia.

\section{Arrhythmia services}

For patients with arrhythmias, clinical guidelines by NHS England, with input and endorsement of BCS and BHRS, recommend that patients with uncontrolled atrial fibrillation (AF) or ventricular tachycardia (VT) will require hospital admission but all elective admissions should be deferred. Urgent pacemaker implantations should continue in patients with symptomatic complete heart block, Mobitz II atrioventricular (AV) block, high grade AV block with symptoms or severe symptomatic sinus node dysfunction with long pauses. Most elective pacemaker generator replacements can be postponed but urgent procedures should continue, especially in pacemaker-dependent patients without underlying rhythm and end of life battery status. Similarly, with regard to complex pacing (defibrillators, cardiac resynchronisation therapy), only urgent implants will be carried out, for example for secondary prevention of sudden cardiac death in cardiac arrest survivors or in patients with VT associated with syncope. Remote follow-up and monitoring of devices should be considered in patients if this is not already enabled and will be led by local hospital protocols. Invasive treatment with ablation should continue for emergency life-threatening tachy-arrhythmias like
AF rapidly conducted via accessory pathway in patients with WolffParkinson-White syndrome, VT storm resistant to pharmacotherapy in a haemodynamically compromised patient, and any other incessant arrythmias (supraventricular tachycardia, AF, atrial flutter) refractory to medical management or associated with significant left ventricular systolic dysfunction and heart failure.

\section{Angiotensin-converting enzyme inhibitors}

It has been shown that the SARS-CoV-2 interacts with the renin-angiotensin-aldosterone system (RAAS) through angiotensin-converting enzyme 2 (ACE2), which is an enzyme that physiologically decreases RAAS activation. It also acts as a receptor for the SARS-CoV-2 virus. ${ }^{2}$ Concerns have been expressed regarding the role of $A C E$ inhibitors and angiotensin receptor blockers (ARBs) in COVID-19, based on the finding that they may increase expression of ACE2. Vaduganathan et a ${ }^{3}$ have discussed the complex area of renin-angiotensin-aldosterone-system (RAAS) inhibition in the setting of COVID-19 and propose an alternative hypothesis suggesting that RAAS blockade may be beneficial rather than harmful in patients with lung injury. The BCS and 
other specialist societies have recommended that patients (eg those with hypertension, heart failure or prior MI) should continue their ACE and ARB inhibitors unless directly advised to stop by a medical practitioner, due to the risk of decompensation following discontinuation of ACE/ARB inhibitors. ${ }^{4}$

\section{Heart failure services}

The BCS has also issued email guidance regarding prioritisation of heart failure service provision during COVID-19 aimed at reducing face-to-face contact and assessing those with greatest clinical need according to NICE guidance, ${ }^{5}$ which recommends the urgent referral of people with suspected heart failure and an NT-proBNP level above $2,000 \mathrm{ng} /$ litre for specialist assessment and transthoracic echocardiography within 2 weeks. There will, however, be challenges in obtaining serum BNP levels in the community, as community phlebotomy services are affected and most general practices have switched to telephone appointments.

\section{The patient with COVID-19 symptoms who has cardiovascular disease}

The presence of this pandemic and its serious consequences for patients and healthcare staff makes this condition foremost in our minds at the current time. The clinical presentation is quite variable, with Guan et al ${ }^{6}$ showing presentations of fever $(88.7 \%)$, cough $(67.8 \%)$, fatigue $(38.1 \%)$, sputum production $(33.4 \%)$ and shortness of breath $(18.6 \%)$ in their Chinese study. Auscultation of the chest should be undertaken with appropriate personal protective equipment (PPE) and where possible the patient should wear a mask to reduce the risk of transmission. The stethoscope should be thoroughly cleaned using appropriate alcohol-based disinfectants.

We must be aware of the diagnostic problems associated with cognitive bias that could potentially occur and thus miss other conditions. A case from our unit is briefly presented in this regard.

A 75-year-old man with a history of angina, hypertension and chronic obstructive pulmonary disease (COPD) presented with worsening shortness of breath. He was febrile, lethargic, confused and vacant with a respiratory rate of 40 , crackles and a systolic murmur. He was treated for an infective exacerbation of COPD and suspected COVID-19. He received antibiotics, steroids, diuretics and non-invasive ventilation. COVID-19 swab was negative. Transthoracic echocardiography showed the presence of critical aortic stenosis for which he underwent aortic valve replacement.

\section{The patient with COVID-19 and pre-existing cardiovascular disease}

Li et $a l^{7}$ in their meta-analysis of six studies inclusive of 1,527 COVID-19 patients reported the prevalence of hypertension and CVD to be $17.1 \%$ and $16.4 \%$, respectively. It has also been suggested that there is a higher risk for adverse events in patients with CVD who contract COVID-19. Case-fatality rates in an analysis of 44,672 confirmed COVID-19 cases from Wuhan, China were noted to be $10.5 \%$ in patients with CVD and $6 \%$ in patients with hypertension, all notably higher than the overall case-fatality rate of $2.3 \% .{ }^{8}$ Physicians managing these patients need to be aware of the likelihood of more severe forms of illness, greater intensive care requirement and worse outcomes.

Brugada syndrome, a rare inherited cardiac condition (channelopathy) associated with increased risk of sudden death, may be unmasked by fever associated with COVID-19. ${ }^{9}$ Clinicians



Fig 1. Brugada syndrome may be unmasked by fever in COVID-19.

should recognise the characteristic ECG pattern (Fig 1), as the diagnosis has implications for immediate and future management, with patients requiring prompt treatment of fever, consideration of hospital admission and cardiac monitoring, and avoidance of the contraindicated drugs listed on www.brugada.org. The Association for Inherited Cardiac Conditions has provided guidance for patients with Brugada syndrome and other inherited cardiac conditions including long QT syndrome, dilated cardiomyopathy, arrhythmogenic cardiomyopathy and hypertrophic cardiomyopathy. ${ }^{10}$

\section{The patient with COVID-19 and cardiovascular complications}

\section{Heart failure}

Zhou et $a{ }^{11}$ reported that $23 \%$ of patients with confirmed COVID-19 infection had evidence of heart failure. Patients with heart failure were also less likely to survive hospitalisation compared to those that did not $(51.9 \%$ vs $11.7 \%)$. It is worth noting that in this series, it is unclear whether the heart failure observed was an exacerbation of pre-existing left ventricular systolic dysfunction, a new cardiomyopathy secondary to myocarditis, a cytokineinduced cardiomyopathy or a combination of these factors. Unproven strategies for cytokine storm include steroids, intravenous immunoglobulin and selective cytokine blockade drugs. There is a higher likelihood of the need for mechanical ventilation in those patients with systolic impairment and reduced ejection fraction. ${ }^{12}$ Treatment is as standard but advanced circulatory support is unlikely to be given due to uncertainty regarding prognosis.

\section{Arrhythmias}

Cardiac arrhythmias are common in patients with COVID-19. In the report from Wuhan, China, arrhythmias occurred in $16.7 \%$ of hospitalized and $44.4 \%$ of ICU patients, although they did not specify which arrhythmias. ${ }^{13}$ Extensive inflammatory reaction plays a major role as it may directly affect the electrical system of the heart 
or facilitate arrhythmias secondary to myocarditis, heart failure and acute coronary syndrome. ${ }^{14}$ In patients with confirmed COVID-19 infection with an indication for permanent pacing, implantation is generally postponed until infection is cured. In unstable cases, a temporary externalised pacing system or a permanent pacemaker can be considered in a dedicated 'hot' theatre for COVID-19 patients following individual assessment of risks versus benefits.

In the setting of cardiac arrest, the gold standard is full aerosolgenerating procedures PPE, but a risk assessment can be done to decide on the level of protection. The Resuscitation Council UK has provided guidance for resuscitation in health care settings. ${ }^{15}$

\section{Cardiac biomarkers (high-sensitivity troponin and BNP/NT-proBNP)}

Detectable high-sensitivity troponin-I (hs-cTnI) has been observed in most patients with COVID-19 and was significantly elevated in more than half of the patients that died..$^{11} \mathrm{~A}$ rise and/or fall of hs-cTn is insufficient to make a diagnosis of acute myocardial infarction (MI). The American College of Cardiology recommends only measuring troponin if there is clinical suspicion of MI. ${ }^{16}$ Primary PCI is the default strategy for STEMI during the pandemic but thrombolysis, while not the preferred option, is recommended to be considered on an individual basis for unstable patients with COVID-19 pneumonia who develop STEMI.?

As with hs-cTnI, patients with COVID-19 often demonstrate significant elevation of BNP or NT-proBNP. The significance of this finding is uncertain and therefore should not necessarily trigger an evaluation or treatment for heart failure unless there is clear clinical evidence to support the diagnosis. Transthoracic echocardiography (TTE) is the investigation of choice in patients with COVID-19 patients and high clinical suspicion for heart failure. BSE guidelines recommend that TTE requests should be approved by a cardiology consultant and a limited bedside study can be performed by a FIT-tested echocardiographer with full PPE to minimise patient exposure and transmission of disease.

Another indication for transthoracic echocardiography is the development of haemodynamic instability, which may be due to cardiac tamponade. This could, for example, be as a result of haemorrhagic conversion of an underlying serous effusion. Transoesophageal echocardiography (TOE) is considered an aerosolgenerating procedure and hence not recommended routinely but can be considered in rare instances (eg infective endocarditis) following a multidisciplinary team discussion where surgery is contemplated.

\section{Research}

As our patients become involved in clinical trials investigating treatments for COVID-19 infection, it needs to be remembered that some of the potential drugs have significant cardiac side effects. Antiviral drugs like lopinavir and ritonavir may cause QT and PR prolongation, while chloroquine and hydroxychloroquine have the potential for myocardial toxicity and pro-arrhythmic effects including AV block, QT prolongation and ventricular arrhythmias.

\section{Summary}

The key messages for non-cardiology physicians are that cardiologists are using alternative means of communication such as electronic media for essential referrals and are postponing any non-urgent procedures including non-urgent echocardiography and cardiac catheterisation. Patients with cardiovascular disease are at increased risk for COVID-19 and also have more severe disease and worse outcomes. Our health system will thus have to gear up its infrastructure for a higher involvement of technology in healthcare delivery.

\section{References}

1 NHS England and NHS Improvement. Clinical guide for the management of cardiology patients during the coronavirus pandemic. NHS England, 2020. Available from www.england.nhs.uk/coronavirus/wp-content/uploads/sites/52/2020/03/specialty-guide-cardiolgy-coronavirus-v1-20-march.pdf [Accessed 20 March 2020].

2 Hoffmann M, Kleine-Weber H, Schroeder S et al. SARS-CoV-2 cell entry depends on ACE2 and TMPRSS2 and is blocked by a clinically proven protease inhibitor. Cell 2020;181:271-80.

3 Vaduganathan M, Vardeny O, Michel T et al. Renin-angiotensinaldosterone system inhibitors in patients with Covid-19. N Engl J Med 2020;382:1653-9.

4 British Cardiovascular Society and British Society for Heart Failure. $B S H \& B C S$ Joint statement on ACEi or ARB in relation to COVID19. BCS, 2020. Available from www.britishcardiovascularsociety. org/news/ACEi-or-ARB-and-COVID-19.

5 National Institute of Health and Care Excellence. Chronic heart failure in adults: diagnosis and management. NICE, 2018. Available from www.nice.org.uk/guidance/ng106/resources/chronic-heart-failure-in-adults-diagnosis-and-management-pdf-66141541311685.

6 Guan WJ, Ni ZY, Hu Y et al. Clinical characteristics of coronavirus disease 2019 in China. N Engl J Med 2020, in press (DOI: 10.1056/ NEJMoa2002032).

7 Li B, Yang J, Zhao F et al. Prevalence and impact of cardiovascular metabolic diseases on COVID-19 in China. Clin Res Cardiol 2020, in press (DOI: 10.1007/s00392-020-01626-9).

8 Wu Z, McGoogan JM. Characteristics of and important lessons from the coronavirus disease 2019 (COVID-19) outbreak in China: Summary of a report of 72314 cases from the Chinese Center for Disease Control and Prevention. JAMA 2020, in press (DOI: 10.1001/jama.2020.2648).

9 Chang D, Saleh M, Garcia-Bengo Y et al. COVID-19 infection unmasking Brugada syndrome. HeartRhythm Case Rep 2020, in press (DOI: 10.1016/j.hrcr.2020.03.012).

10 Association for Inherited Cardiac Conditions. COVID-19. AICC, 2020. Available from https://theaicc.org/?page_id=649 [Accessed 14 April 2020]

11 Zhou F, Yu T, Du R et al. Clinical course and risk factors for mortality of adult inpatients with COVID-19 in Wuhan, China: a retrospective cohort study. Lancet 2020;395:1054-62.

12 Li SS, Cheng C, Fu C et al. Left ventricular performance in patients with severe acute respiratory syndrome: 30 day echocardiographic follow up study. Circulation 2003;108:1798-803.

13 Wang D, Hu B, Hu C et al. Clinical characteristics of 138 hospitalized patients with 2019 novel coronavirus-infected pneumonia in Wuhan, China. JAMA 2020, in press (DOI: 10.1001/jama.2020.1585).

14 Madjid M, Safavi-Naeini P, Solomon SD, Vardeny O. Potential effects of coronaviruses on the cardiovascular system; a review. JAMA Cardiol 2020, in press (DOI: 10.1001/jamacardio.2020.1286).

15 Resuscitation Council UK. March 2020. COVID-19 resources: healthcare settings. Available from www.resus.org.uk/media/statements/resuscitation-council-uk-statements-on-covid-19-coronavirus-cpr-and-resuscitation/covid-healthcare-resources.

16 Januzzi JL. Troponin and BNP use in COVID-19. Cardiology Magazine, 18 March 2020.

Address for correspondence: Prof Gershan Davis, School of Medicine and Dentistry, University of Central Lancashire, Preston PR1 2HE, UK.

Email: gdavis1@uclan.ac.uk 